\title{
A nonlinear model for the characterization and optimization of athletic training and performance
}

\author{
James D. Turner, Michael J. Mazzoleni, Jared A. Little, Dane Sequeira, Brian P. Mann \\ Dynamical Systems Research Laboratory, Department of Mechanical Engineering and Materials Science, Duke \\ University, Durham, NC, USA
}

\section{Summary}

Study aim: Mathematical models of the relationship between training and performance facilitate the design of training protocols to achieve performance goals. However, current linear models do not account for nonlinear physiological effects such as saturation and over-training. This severely limits their practical applicability, especially for optimizing training strategies. This study describes, analyzes, and applies a new nonlinear model to account for these physiological effects.

Material and methods: This study considers the equilibria and step response of the nonlinear differential equation model to show its characteristics and trends, optimizes training protocols using genetic algorithms to maximize performance by applying the model under various realistic constraints, and presents a case study fitting the model to human performance data.

Results: The nonlinear model captures the saturation and over-training effects; produces realistic training protocols with training progression, a high-intensity phase, and a taper; and closely fits the experimental performance data. Fitting the model parameters to subsets of the data identifies which parameters have the largest variability but reveals that the performance predictions are relatively consistent.

Conclusions: These findings provide a new mathematical foundation for modeling and optimizing athletic training routines subject to an individual's personal physiology, constraints, and performance goals.

\section{Keywords: Training - Fitness - Fatigue - Dynamical systems - Optimization}

\section{Introduction}

Training is widely accepted as a method to improve one's performance in sports. However, athletes typically have to rely on experience, heuristics, and rough approximations to design their training routines. The current models available for predicting performance as a function of training have significant predictive limitations. A more sophisticated model is needed to improve performance predictions and design optimal training strategies.

Banister proposed a mathematical model that was based on a system of linear ordinary differential equations to quantify the effect of training on performance for collegiate swimmers $[3,7]$. The model accounted for two primary physiological components: the positive effects of training, called fitness, and the negative effects of training, called fatigue. Several studies have built upon the Banister model with promising results $[5,6,8,10,12,15]$. However, these studies also recognized that the Banister model was based on linear systems theory, which limits its accuracy and applicability. One concerning feature from past linear performance models is that they predict steadystate performance continues to increase indefinitely with increases in training stress [12]. Thus linear performance models only capture the temporal or short-term negative effects on performance and not the important long-term and nonlinear relationships that limit performance. The result is that optimal performance cannot be determined from a linear performance model since more training always results in a prediction of better performance. One of the important aspects of the present work is the introduction of a generic mathematical framework to capture a diminishing rate of return, or performance saturation, and over-training. It is the incorporation of these well-known phenomena into a performance modeling framework that provides a more realistic and necessary counter balance; the inclusion of these phenomena also allows performance to be optimized. However, despite the potential concerns associated with the linear modeling approach, it has been 
used to successfully inform training strategies for many athletes by approximating optimal recovery times between workouts, predicting the success of training regimens, and determining how an athlete should taper before a competition $[5,7,8,10,12,13,15]$.

Some researchers have incorporated nonlinear elements with Bannister's linear model to improve prediction capabilities $[4,9,16,17]$. Hellard et al. incorporated a Hill function as a scaling term for stress to model a threshold in the stress-response relationship [9]. Busso introduced an additional state variable to model the increased effect on fatigue of more frequent stresses [4], and his model was further studied by Thomas et al. [16, 17].

This article differentiates itself from prior work by presenting a novel refinement of Banister's model that incorporates the nonlinear effects of saturation and overtraining without introducing additional state variables or auxiliary functions. This enables the model to account for nonlinear physiological effects while maintaining an intuitive and simple mathematical structure. The proposed model can also be used for designing optimal training strategies based on the personal physiology, constraints, and performance goals of an individual.

\section{Material and methods}

This section introduces the novel nonlinear performance model, explores how it can be applied to optimize training strategies to achieve performance goals under various realistic constraints, and describes the experimental testing strategy.

Many past works have sought to model the effect of training on performance with a set of linear ordinary differential equations to describe both the positive effects, sometimes called fitness $f$, and negative effects, sometimes called fatigue $u$, of training on performance. While changes in both fitness and fatigue can be viewed as the sum of various muscular, psychological, and nutritional factors, these models consider changes in fitness and fatigue due only to training. Performance can then be determined from these values as the difference between fitness and fatigue, $p=p_{0}+f-u$, where $p_{0}$ is an individual's performance in an untrained state [7]. While short-term performance can often be predicted from this approach, it is impossible to find the training stress that results in an optimal equilibrium performance. Predictions made using this linear modeling approach indicate that equilibrium performance indefinitely improves with increases in training stress, i.e. saturation and the negative effect of overtraining are not captured.

The original performance modeling concept can be modified to the following set of nonlinear differential equations to capture the effect of training on performance

$$
\begin{aligned}
& \dot{f}+\frac{1}{\tau_{1}} f^{\alpha}=k_{1} \sigma, \\
& \dot{u}+\frac{1}{\tau_{2}} u^{\beta}=k_{2} \sigma,
\end{aligned}
$$

where $f$ is fitness as a function of time, $u$ is fatigue as a function of time, $\sigma$ is the training stress impulse as a function of time, $\tau_{1}$ and $\tau_{2}$ are time constants, $k_{1}$ and $k_{2}$ are gain terms, $\alpha$ and $\beta$ are exponents that represent the model's nonlinearities, and $t$ is time. Note that an overdot indicates a time derivative. The parameters are personspecific constants that depend on various physiological factors and can be determined from performance tests and parameter estimation algorithms.

It is important to note that the linear Banister model can be recovered from Eqs. (1) and (2) by setting $\alpha=\beta=1$, which results in the form of the linear Banister model proposed by Busso [6] or, with $\tau_{1}=k_{1}^{-1}, \tau_{2}=k_{2}^{-1}$, and $p_{0}=0$, the original model proposed by Banister [3]. In essence, the introduction of nonlinearity enables additional phenomena, such as saturation and over-training, to be captured while still accounting for increases and decreases in performance due to training.

\section{Constant daily training stress}

The special case of a time invariant or constant daily training stress is useful conceptually to illustrate how Eqs. (1) and (2) capture the effects of training saturation and over-training. For the results that follow, the parameter values given in Table 1 were used. Note that these values were chosen arbitrarily to produce a realistic response, but the model parameters are dependent on an individual's physiological characteristics.

If a constant daily training stress is applied, performance will eventually stagnate or plateau. This case can be explored from the equilibria of Eqs. (1) and (2) when $\dot{f}=\dot{u}=0$. The steady-state performance can then be obtained analytically and becomes

$$
\tilde{p}=p_{0}+\left(k_{1} \tau_{1} \sigma\right)^{1 / \alpha}-\left(k_{2} \tau_{2} \sigma\right)^{1 / \beta} .
$$

The stress that causes the maximum performance is found from $\mathrm{d} \tilde{p} / \mathrm{d} \sigma=0$, which gives

$$
\sigma_{1}=\left(\left(\frac{\beta}{\alpha}\right)^{\alpha \beta} \frac{\left(k_{1} \tau_{1}\right)^{\beta}}{\left(k_{2} \tau_{2}\right)^{\alpha}}\right)^{1 /(\beta-\alpha)} .
$$

Another important stress value is the training stress that would result in no performance improvement. This value is obtained by equating the equilibria of Eqs. (1) and (2) to obtain the following expression

$$
\sigma_{2}=\left(\frac{\left(k_{2} \tau_{2}\right)^{\alpha}}{\left(k_{1} \tau_{1}\right)^{\beta}}\right)^{1 /(\beta-\alpha)} .
$$


Table 1. Parameter values and initial conditions for simulations and examples. The units for performance, stress, and time are arbitrary, but the parameter values presented in this table correspond to reasonable values using days for [time], BikeScore [14] for [stress], and a cyclist's maximum possible average power output in Watts over a 10-minute interval for [performance]. The parameters $f_{0}$ and $u_{0}$ are the initial conditions for fitness and fatigue, respectively

\begin{tabular}{ccc}
\hline Parameter & Value & Units \\
\hline$\tau_{1}$ & 61 & [performance] $^{\alpha-1}[$ time] \\
$\tau_{2}$ & 5.5 & [performance] $^{\beta-1}[$ time] \\
$\alpha$ & 1.16 & - \\
$\beta$ & 0.85 & - \\
$k_{1}$ & 0.10 & [performance] $\left[\right.$ time] $^{-1}[$ stress] \\
$k_{2}$ & 0.12 & [performance] $\left[\right.$ time] $^{-1}[$ stress] \\
$p_{0}$ & 155 & [performance] $^{-1}$ \\
$f_{0}$ & 70.9 & [performance] \\
$u_{0}$ & 24.5 & [performance]
\end{tabular}

The steady-state trends for fitness, fatigue, and performance are shown in Figs. 1 and 2. These plots show several interesting aspects. First, there are diminishing returns in performance as training stress is increased. Performance continues to increase along with increases in training stress until $\sigma_{1}$ is reached. If training stress is increased beyond $\sigma_{1}$, then over-training occurs and performance deteriorates as more training stress will have a negative impact on training performance.

The results shown in Figs. 1 and 2 only provide the steady-state training and performance relationships. However, Fig. 3 compares the temporal evolution of

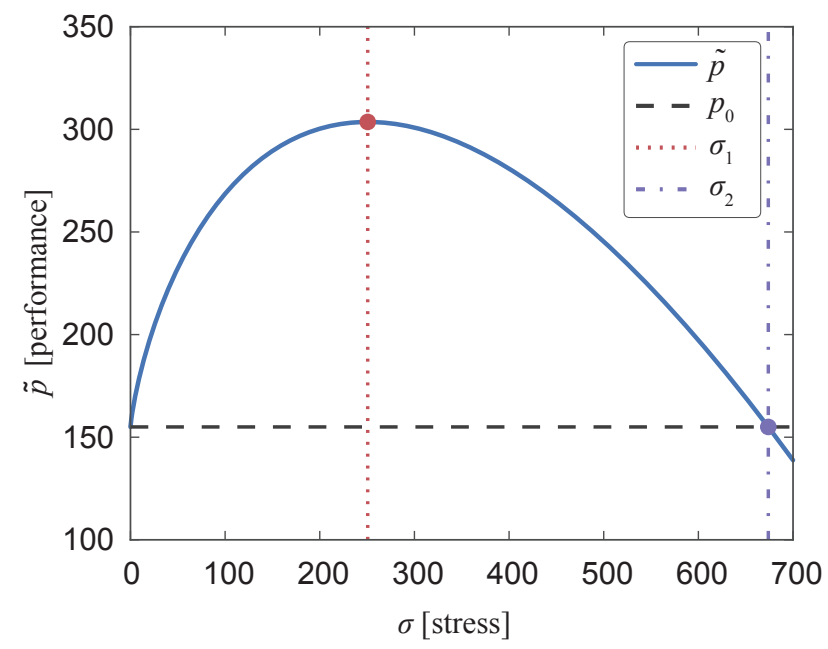

Fig. 1. Plot illustrating how the nonlinear performance model captures the physiological phenomena of saturation and over-training. For this plot, the special case of a constant daily training stress is considered performance for the constant daily training stresses of $\sigma_{1}$ and $\sigma_{2}$. This figure illustrates both the super-compensation effect and a plateau in performance after a sufficient period of time.

The analysis presented in this section has only considered the case of a constant daily training stress to provide a simple example that highlights the ability of the nonlinear performance model to capture saturation effects and over-training. However, the more general case, where $\sigma$ is allowed to vary with time, is of greater interest. Allowing $\sigma$ to vary as a function of time more accurately describes practical scenarios in which an individual's training schedule changes on a daily basis. Furthermore, since the nonlinear performance model captures realistic phenomena, such as saturation and over-training, the model

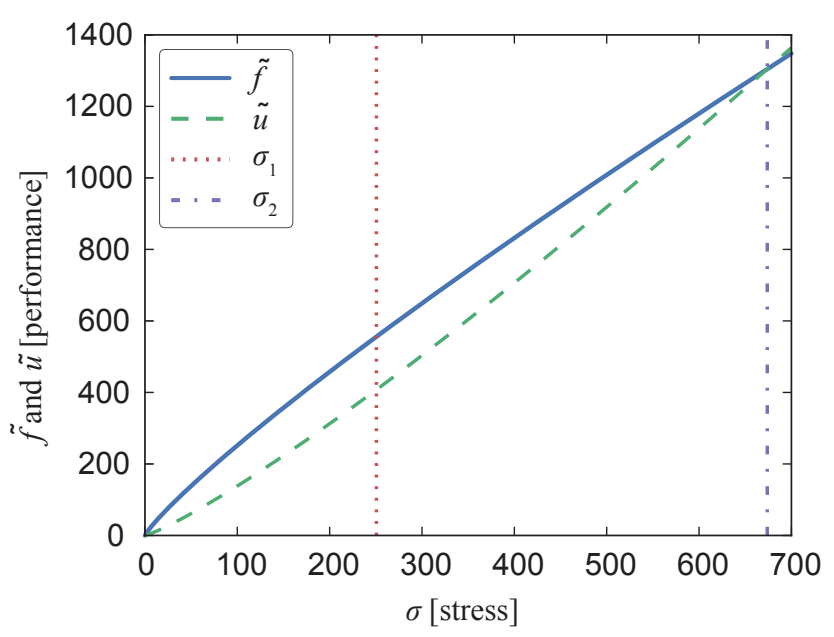

Fig. 2. Plot that depicts the compounding effects of fatigue and diminishing returns of fitness gains with increases in training stress. Maximum performance is achieved when $\tilde{p}=p_{0}+\tilde{f}-\tilde{u}$ is maximized, and no performance gains are achieved when $\tilde{f}=\tilde{u}$

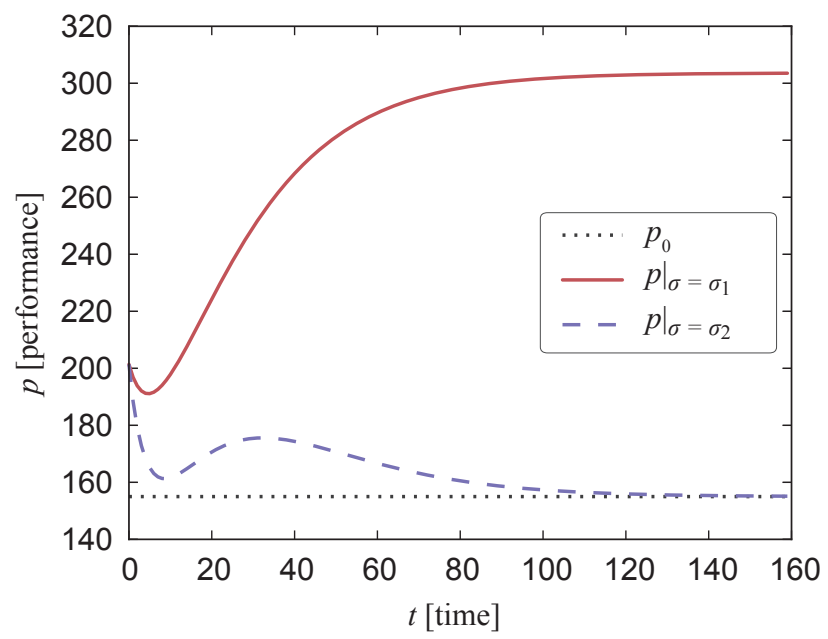

Fig. 3. Plot illustrating the super-compensation effect and plateau in performance for the special case of a constant daily training stress 
can be used to predict performance and design an optimal training routine. Thus, the next section considers different scenarios where $\sigma(t)$ can be varied to optimize performance for athletes based on their personal physiological characteristics, constraints, and performance goals.

\section{Constrained optimized training}

The constant daily training stress that was investigated in the previous section is useful for understanding the model conceptually, but it is not necessarily optimal for use as a training tool. The typical training strategy that athletes employ to prepare for an event is a period of base training with progressively increasing training intensity, followed by a build-up phase at relatively high intensity, and then finishing with a taper phase to reduce fatigue on the competition date. This strategy helps the individual to increase their fitness gradually to avoid injury while maximizing performance on race day.

Depending on an individual's personal physiological characteristics, constraints, and performance goals, the optimal training program can look very different. To explore how the nonlinear model can be applied to optimize a training program and to illustrate the influence of various constraints, this section explores a specific scenario: the case of optimizing the daily training stresses for twelve consecutive weeks of training to maximize performance for a cycling race on the $85^{\text {th }}$ day. The parameter values that were kept constant during the optimization process are provided in Table 1 . The optimization was performed using genetic algorithms, which are heuristic optimization algorithms based on the idea of natural selection $[2,11]$. Genetic algorithms are a popular optimization method for nonlinear problems. The genetic algorithms used tournament selection, BLX- $\alpha$ crossover, Gaussian mutation, external penalty functions to enforce constraints, and were run ten times for each case studied to ensure consistent results.

\section{Constant daily training stress constraint with tapering}

Perhaps the simplest improvement to the constant daily training stress routine described earlier is to allow a short rest period prior to race day. This period of rest or limited exercise is typically referred to as a taper and allows for the dissipation of accumulated fatigue, thus improving final performance. The considerably shorter time constant of the fatigue $\tau_{2}$, relative to that of the fitness $\tau_{1}$, results in improved performance after short rest periods where quick recovery from fatigue outweighs slower losses in fitness. The simplest taper scheme is to stop training a few days before the end of the season. Figure 4 illustrates an example of this approach, with a notable increase in performance due to tapering. To compare the results with the previously described constant daily stress routine, two scenarios were considered: 1 ) using the value of $\sigma_{1}$ found

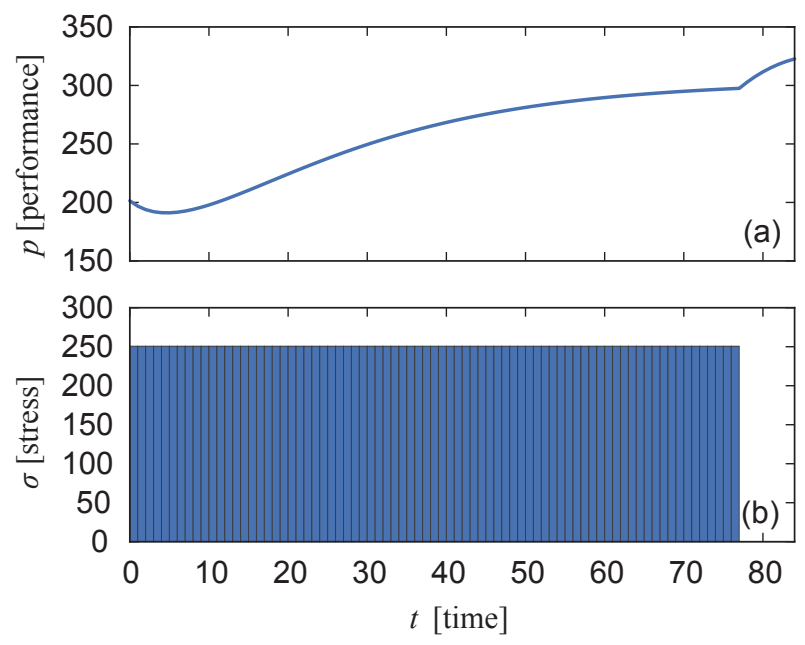

Fig. 4. Plots showing (a) performance and (b) daily training stress for a 7-day taper. The benefit of the taper can be observed by examining the considerable increase in performance following the onset of the taper

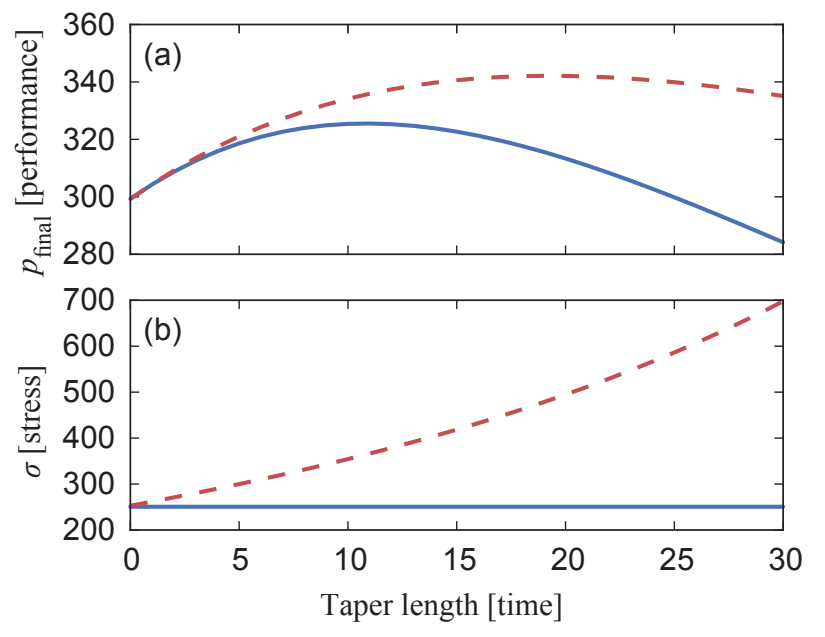

Fig. 5. Plots showing (a) final performance and (b) constant daily training stress for various taper lengths for two cases: 1) the constant daily training stress $\sigma_{1}$ calculated by Eq. (4) (solid blue line); and 2) the constant daily training stress that produces the highest performance at the end of the season for a given taper length (dashed red line)

in Eq. (4); and 2) using the stress that produced the highest performance at the end of the season for each taper length. The results of these scenarios can be seen in Fig. 5.

From these results, it is clear that the constant daily training stress scenario is improved by simply implementing a taper. Increasing the length of this taper can improve performance by reducing fatigue, up until the point where the rate of fitness atrophy begins to outweigh the rate of fatigue loss. In the provided example, this optimal taper length is roughly 10 days, as shown by the peak in Fig. 5(a). An additional intriguing result can be observed regarding the case where constant daily training stress 
leading up to the taper was optimized to maximize final performance for each taper length: allowing constant daily training stress to increase without bound does not result in unbounded performance gains. In this scenario, as taper length increases, larger training stress values are prescribed, as shown in Fig. 5(b). However, the fitness gains due to higher stresses and fatigue reduction due to a longer taper are balanced or outweighed by the increased fatigue due to higher stresses and fitness atrophy due to a longer taper. The result of this effect can be seen in the downturn of the dashed curve in Fig. 5(a).

\section{Uniform weekly schedule constraint}

This section considers the case where an athlete might be constrained by their routine weekly schedule. In particular, we investigated the optimal training schedule when the daily training stresses were allowed to vary within the week, but every week was required to be identical. This creates a periodic training regimen with a seven day period. When relating this uniform weekly schedule to the uniform daily training stress case, the steady-state performance is of interest. The repeated variation within each week creates a periodic steady-state solution. Figure 6 shows the steady-state performance for the uniform weekly schedule that maximizes the average weekly performance in steady-state. These results demonstrate that the average performance value obtained using the uniform weekly schedule constraint is identical to the optimal performance value for the constant daily stress scenario with $\sigma=\sigma_{1}$ from Eq. (4). This indicates that there is not necessarily a unique solution that produces a maximum average performance when prescribing a uniform weekly training schedule constraint.

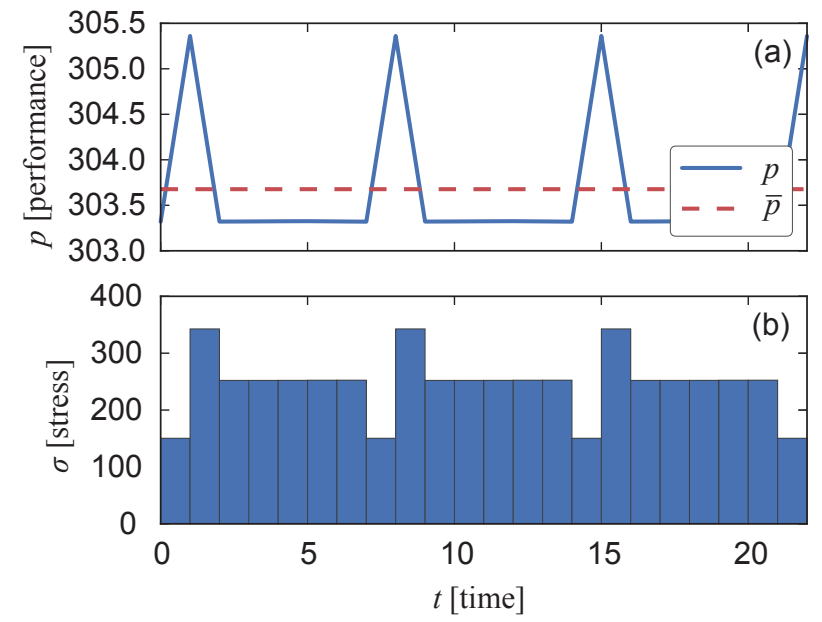

Fig. 6. Plots showing (a) performance $p$ and mean performance $\bar{p}$ and (b) prescribed daily training stress $\sigma$ for the case of a uniform weekly training stress constraint. The results shown occur after the system has reached a steadystate. Three weeks are shown to illustrate the periodic nature of the result

\section{Maximum daily training stress constraint}

From a practical standpoint, an individual only has a limited number of hours to train each day. Thus it is sensible to limit the training stress to a maximum allowable value that could reasonably be performed each day. For this purpose, we used a training stress of $\sigma_{\text {allow }}=300$, which roughly equates to 5-6 hours of training on a bicycle. Figure 7 shows a training routine that results in optimal performance on race day, given a maximum training stress of $\sigma_{\text {allow }}=300$ as the only constraint. From these results, it is interesting to note that the optimal training routine does not contain a build-up in the daily training stress; thus, this constraint alone does not allow for a realistic progression in the training load as fitness improves. The optimal training routine requires training at the maximal allowable training stress every day until the taper begins. In order to develop more realistic training protocols, additional constraints must be considered.

\section{Training load constraints}

The constraints presented in the previous sections demonstrate important aspects of the training-performance relationship, but they do not yield truly realistic training plans. This section considers the notion of a training load constraint, which results in optimal training plans that seek to balance performance gains with injury risk mitigation. The training plans that result from considering these training load constraints begin to resemble the training plans that coaches and traditional wisdom would recommend.

Maximum ATL constraint. Acute training load (ATL) is commonly used by athletes to quantify the short-term effects of fatigue [1]. This section seeks to optimize performance where daily training stress is constrained by both ATL and a maximum daily training stress. ATL can be defined as

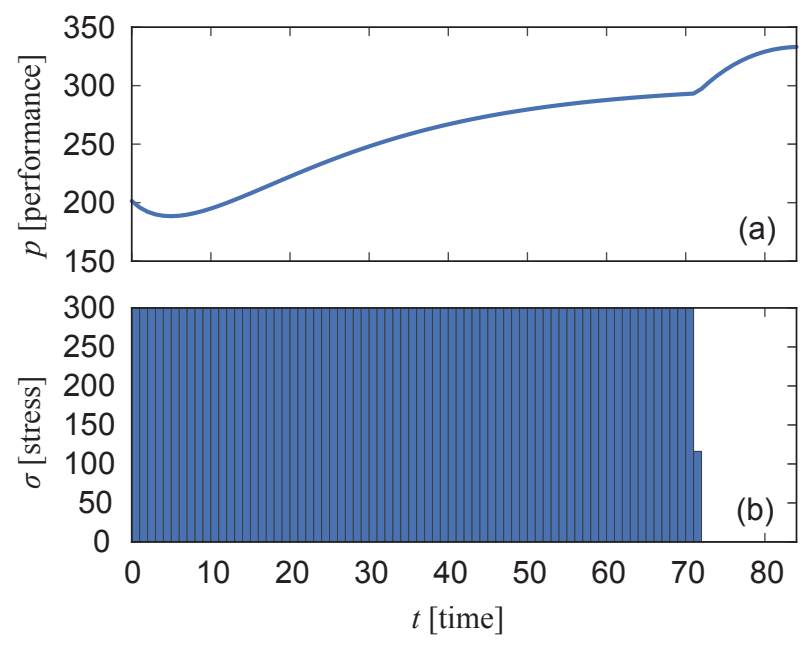

Fig. 7. Plots depicting (a) performance and (b) prescribed daily training stress for the case of a maximum daily training stress constraint $\sigma_{\text {allow }}=300$ 


$$
A_{n+1}=A_{n}+\left(\sigma_{n}-A_{n}\right)\left(1-\mathrm{e}^{-1 / \tau}\right),
$$

where the acute training load $A_{n}$ and the daily training stress $\sigma_{n}$ on the $n^{\text {th }}$ day were used along with the time constant $\tau=7$ days to determine the acute training load $A_{n+1}$ on day $n+1$.

Figure 8 depicts an example where the ATL was constrained to a maximum value of $\mathrm{ATL}_{\text {allow }}=200$ and the daily training stress was constrained to a maximum value of $\sigma_{\text {allow }}=300$. It is interesting to note that the daily training stress is relatively high at the initial stages of training; therefore, the ATL constraint alone does not allow for a progressive build-up in the training load as fitness improves. However, after an initial transient period, the daily training stress is limited throughout the middle stages of training by the ATL constraint and begins to resemble a more realistic prescribed training strategy.

The effect of varying the maximum ATL constraint is investigated in Figure 9. As expected, increasing the maximum ATL constraint increases final performance at a diminishing rate of return, but also increases the risk of fatigue-related injury.

Person-specific fatigue constraint. The ATL metric is a popular standardized metric, but it is not person-specific and is instead based on a generic time constant. An alternative approach is to use a person-specific constraint based on fatigue $u$ from the nonlinear model. For example, the constraint explored in this section is defined as

$$
\sigma_{\text {allow }}=300\left(0.1+0.9 \mathrm{e}^{-u / 800}\right) \text {. }
$$
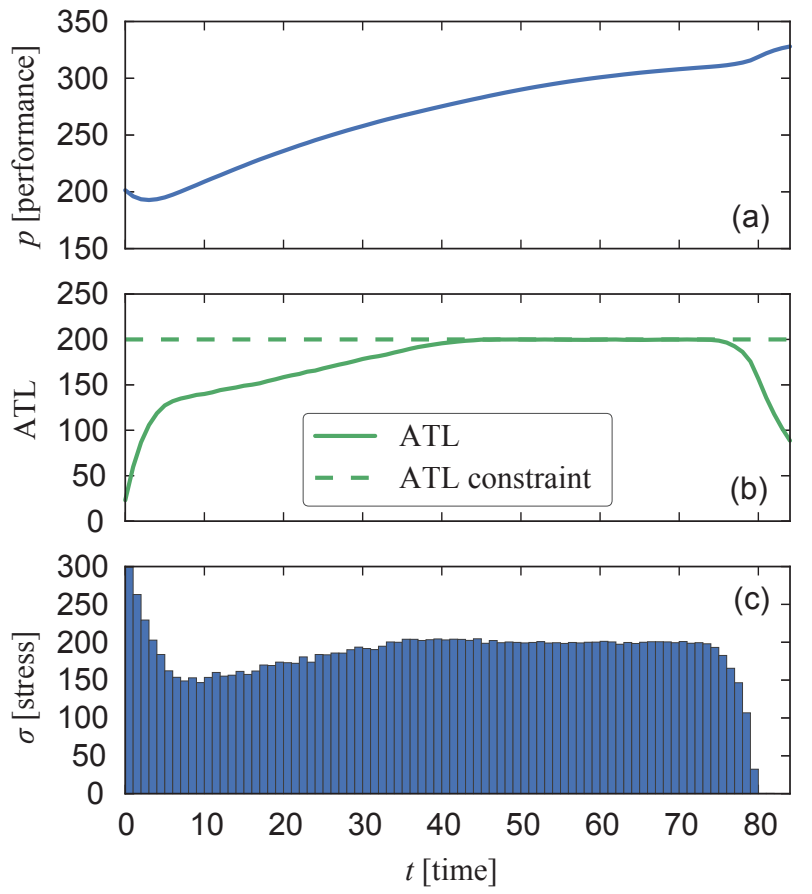

Fig. 8. Plots showing (a) performance, (b) ATL, and (c) daily training stress. The optimization was constrained by $\sigma_{\text {allow }}=300$ and $\mathrm{ATL}_{\text {allow }}=200$

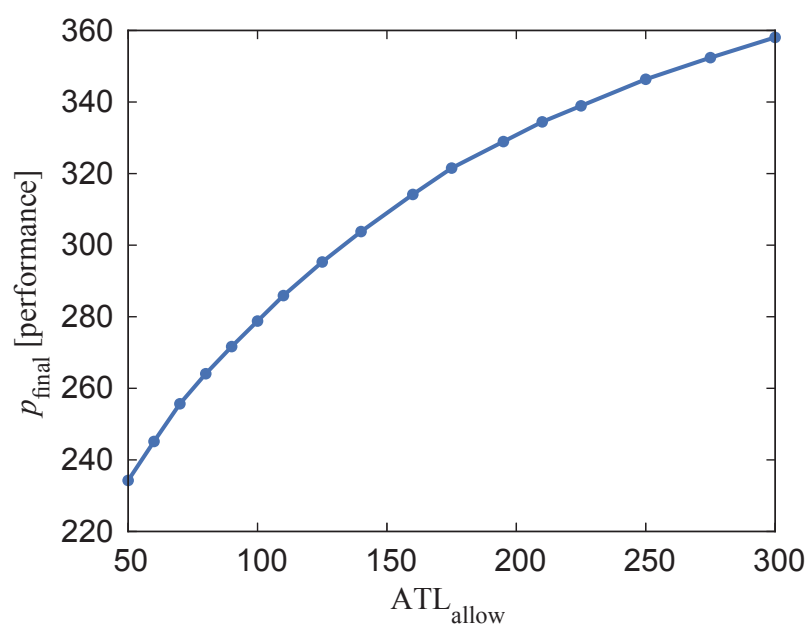

Fig. 9. Plot depicting optimal final performance versus $\mathrm{ATL}_{\text {allow }}$. The maximum daily stress constraint of $\sigma_{\text {allow }}=300$ was held constant

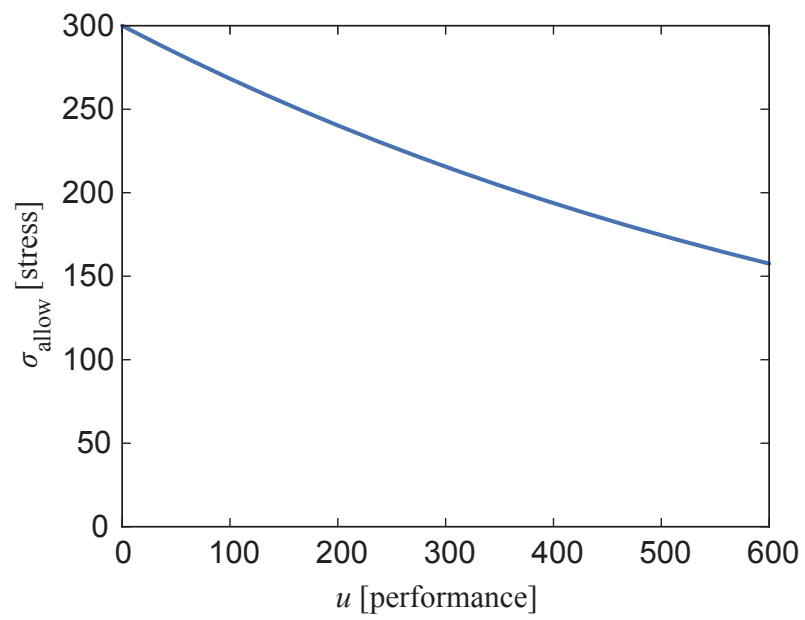

Fig. 10. Plot of the maximum allowable training stress as a function of fatigue described by Eq. (7)

This fatigue-based constraint is depicted in Fig. 10. This constraint allows for fairly high training stress when fatigue is low, but as fatigue increases, the allowable training stress asymptotically decreases.

The resulting optimal training routine and performance are shown in Fig. 11. Similar to the ATL constraint, the stresses are lower throughout the middle of the training program, but there is no progression in training load during the early stages of training. Therefore, these results indicate that an additional constraint is required to produce a progression in the allowable stresses during the earlier stages of training.

Maximum ATL/CTL constraint. Chronic training load (CTL) is often calculated in conjunction with ATL to assess athletic fatigue [1]. While ATL considers the shortterm effects of fatigue, CTL considers the longer-term effects. This section considers a constraint that is based 


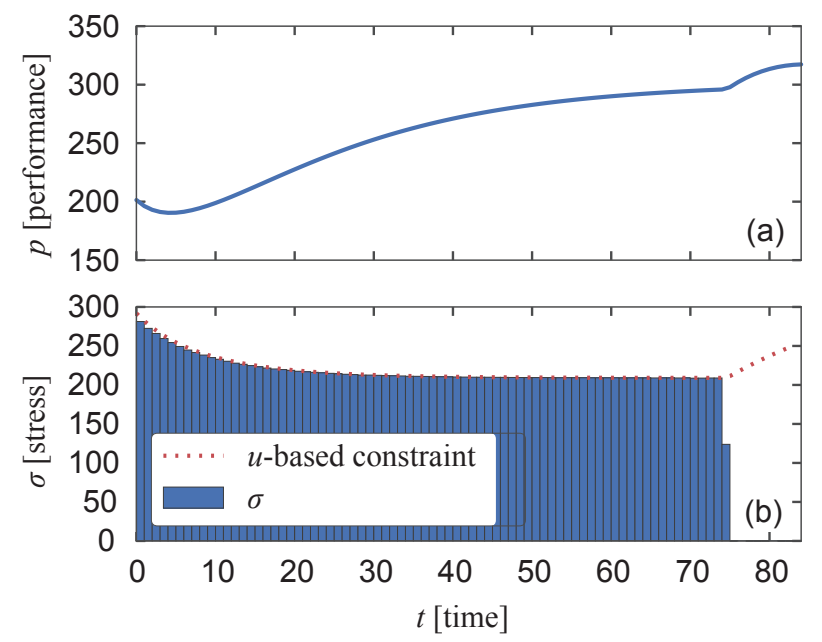

Fig. 11. Plots of (a) performance and (b) training stress when a person-specific fatigue constraint and a daily maximum training stress of $\sigma_{\text {allow }}=300$ were applied

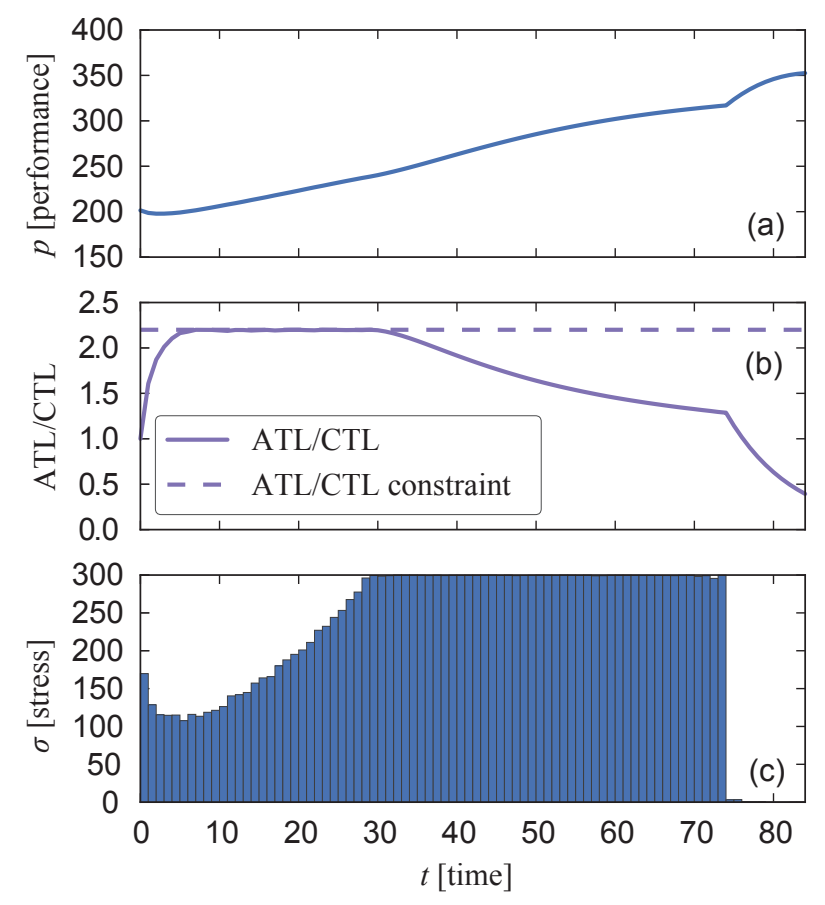

Fig. 12. Plots showing (a) performance, (b) ATL/CTL ratio, and (c) daily training stress as optimized using the ATL/CTL constraint. A daily maximum stress of $\sigma_{\text {allow }}=300$ and $(\mathrm{ATL} / \mathrm{CTL})_{\text {allow }}=2.2$ were imposed as constraints

on the ratio of ATL/CTL. Conceptually, this constraint ensures that a longer period of training sufficiently prepares an individual for more intense short-term training effects. CTL can be calculated as

$$
C_{n+1}=C_{n}+\left(\sigma_{n}-C_{n}\right)\left(1-\mathrm{e}^{-1 / \tau}\right),
$$

where the chronic training load $C_{n}$ and the daily training stress $\sigma_{n}$ on the $n^{\text {th }}$ day were used along with the time

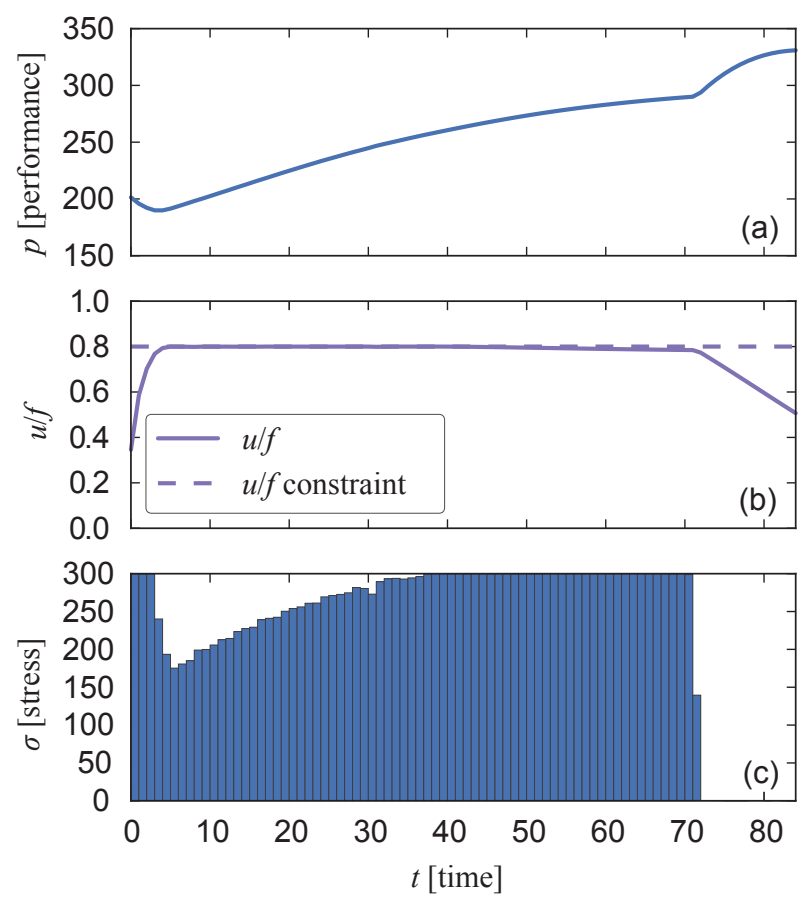

Fig. 13. Plots showing (a) performance, (b) $u / f$, and (c) daily training stress as optimized using the $u / f$ constraint. A daily maximum stress of $\sigma_{\text {allow }}=300$ and $(u / f)_{\text {allow }}=0.8$ were imposed as constraints

constant $\tau=42$ days to determine the chronic training load $C_{n+1}$ on day $n+1$.

An optimal training routine that considers a maximum ATL/CTL constraint is depicted in Fig. 12. It is interesting to note that this constraint does a better job than previous constraints in terms of implementing a progression in training load during the early stages of the training program. However, as shown in this example, the training load during the first few days might still be relatively high; this was true for other ATL/CTL ratios that were also investigated. The training stress during the middle stages of the training program eventually becomes the maximal allowable daily value until the start of the taper.

Person-specific fatigue/fitness constraint. Analogous to the ATL/CTL constraint is a maximum fatigue/fitness constraint, which has the additional benefit of being person-specific. Figure 13 shows that this fatigue/fitness constraint provides somewhat of a progression in the training stress at the onset of a training program. The behavior is similar to the case where the ATL/CTL ratio is used as a constraint. After a relatively large training stress during the first few days of training, the fatigue/fitness ratio forces progression in training stress until reaching the maximum stress of $\sigma_{\text {allow }}=300$, with a taper occurring at the end.

\section{Training progression constraint}

It is common for most training programs to progress the training load during the early stages of training. This 
strategy helps the individual improve their fitness before higher-stress loads are introduced and is often used to avoid injury. This section describes a constraint that improves upon using the ATL/CTL or the $u / f$ ratio. More specifically, this section investigates constraining the training stress as a function of fitness. Intuitively, when fitness is low, the allowable stress should be low to limit the chance of injury, while as fitness increases, the allowable stress should approach a reasonable maximum value. An example of such a relationship is

$$
\sigma_{\text {allow }}=300\left(1-0.9 \mathrm{e}^{-f / 150}\right) .
$$

This constraint is depicted in Fig. 14. The resulting optimal training routine and performance are shown in Fig. 15. As shown, this constraint provides a very nice build-up effect during the early stages of training.

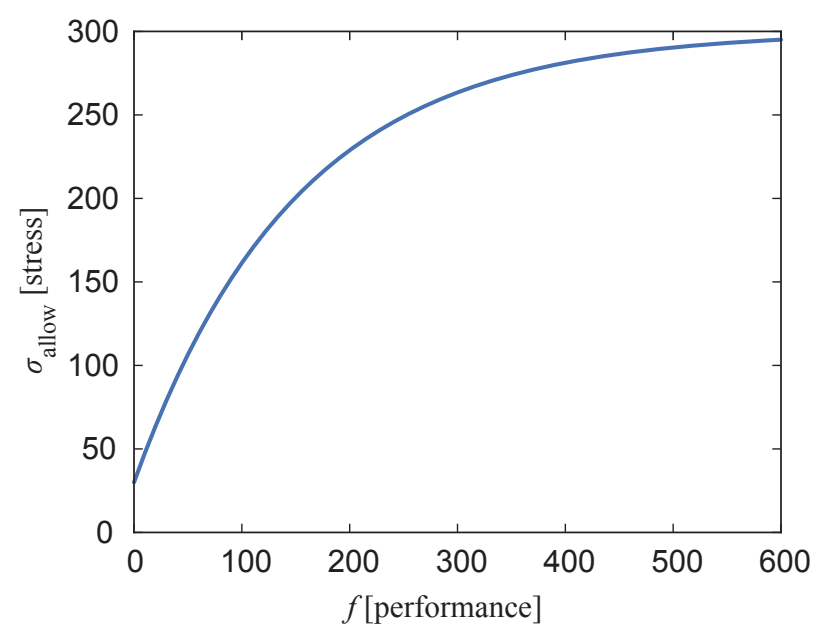

Fig. 14. Plot depicting maximum allowable training stress as a function of fitness described by Eq. (9)

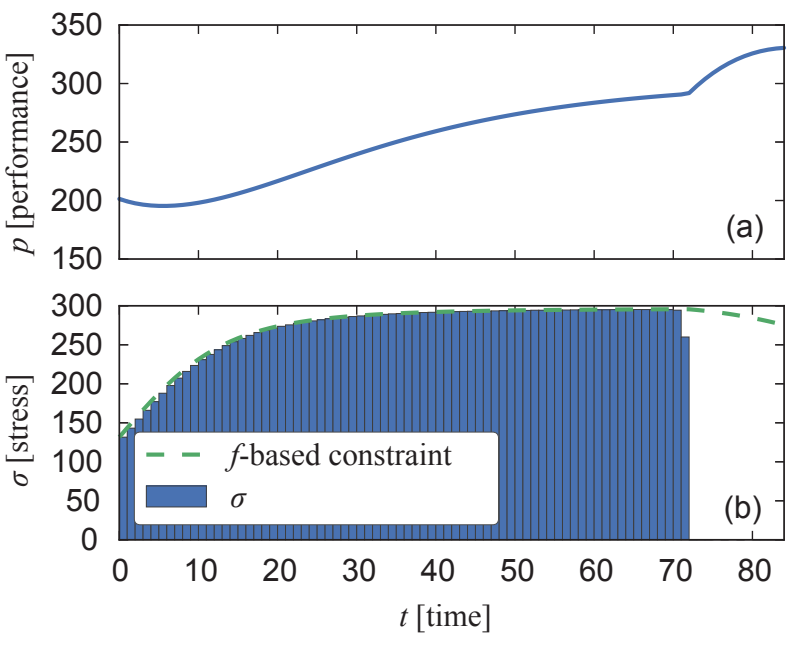

Fig. 15. Plots of (a) performance and (b) daily training stress when a person-specific $f$-based constraint and a daily maximum training stress of $\sigma_{\text {allow }}=300$ were applied

\section{Combination of constraints}

In order to develop truly realistic optimal training routines, a combination of constraints must be applied. For example, the fatigue-based maximum stress constraint illustrated in Fig. 10, the maximum fatigue/fitness ratio constraint illustrated in Fig. 13(b), and the fitness-based maximum stress constraint illustrated in Fig. 14 were applied simultaneously, and the resulting training routine is shown in Fig. 16. This combination of constraints is much more similar to a training program that would be developed by a human coach - it has an initial period of training progression, mostly constant intensity for the middle of the season, and then a reasonable taper period. By incorporating both fitness-based and fatigue-based constraints, the routine attempts to avoid injury from effects of both low fitness and high fatigue. The fatigue/fitness ratio constraint helps to smooth the transition from the training progression at the beginning to the relatively constant intensity for the middle of the season. Out of all the constraint scenarios that were investigated in this study, this combination of constraints generates the most realistic optimal training routine.

\section{Human performance data}

As shown in the previous sections, the nonlinear model can be used to design optimal training programs subject to an individual's personal constraints. However, in order to apply the model in practice, the system parameters must first be estimated based on measurements of an individual's performance. This requires a parameter estimation

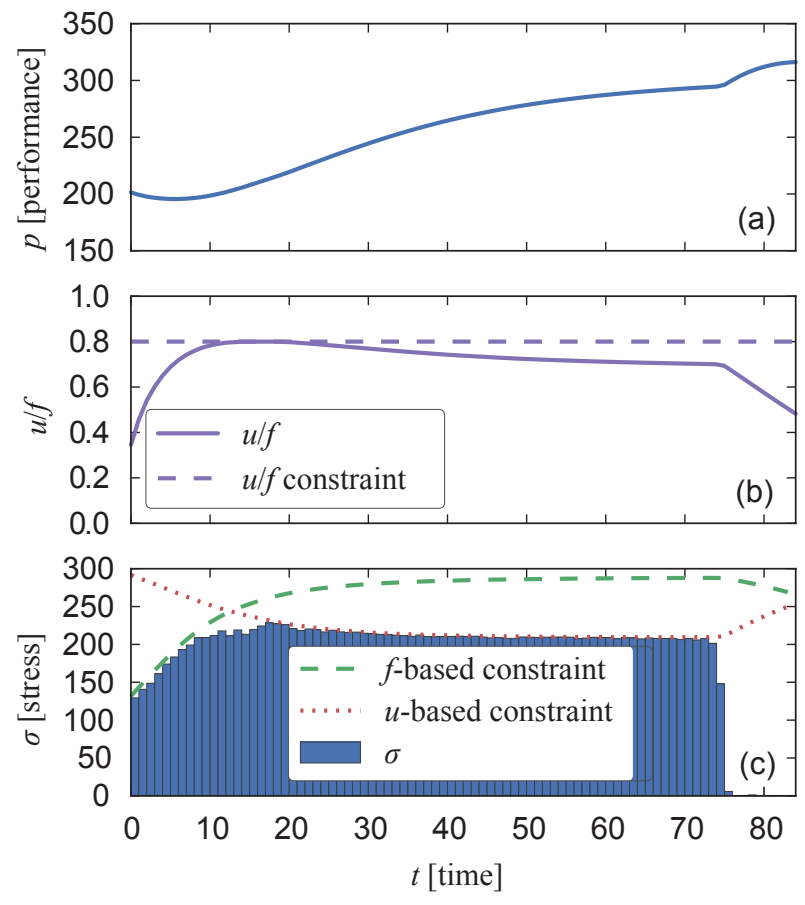

Fig. 16. Plots of (a) performance (b) $u / f$, and (c) daily training stress when using the $u$-based, $f$-based, and $u / f$-based constraints 
algorithm, in this case a genetic algorithm, to optimize the parameters to fit the data $[2,11]$. Parameter estimation was performed on a case study of historical data, and an assessment of the model limitations was performed.

\section{Parameter estimation}

A retrospective study was conducted using historical data from one cyclist in order to assess the parameter estimation strategy and accuracy. Garmin Vector power meter pedals were used to measure the power output produced by the cyclist $(\leq 2 \%$ error). The performance was defined as the cyclist's average power output over the most intense 10-minute interval during each exercise bout, and the training stress was defined using the BikeScore metric [14]. The best fit was estimated using a genetic algorithm with the mean absolute error as the objective function.

Since the analysis was performed on historical data, the cyclist was not necessarily exercising at maximum intensity for any one of the data points, so some performance measurements may have been below the true value. Unfortunately, the historical data included neither measurements of blood lactate and heart rate to quantify the effort level nor the individual's sleep and diet records to assess rest and recovery conditions. However, some knowledge of life events (e.g. illness) was available to eliminate data points that were not properly representative. The resulting data set is shown in Fig. 17.

\section{Uncertainty estimation}

To determine the predictive limitations of the model, an assessment was performed based on cross-validation. This involved fitting the model to subsets of the data and then evaluating how well it applied to the remaining data. Of the 18 experimental trials, 9 unique trials were randomly selected and the parameters were fit to those 9 points. This process of randomly selecting 9 unique trials, one for each degree of freedom, and fitting the parameters was repeated 333 times in total to estimate the distribution of all possible sets of 9 unique trials. Using the parameters from the randomly selected trials and the initial conditions from the best fit to all trials, the model was integrated for the entire time period.

\section{Results}

The results showed that the model's performance predictions fit the experimental data with small error, and fitting to random subsets of data showed that some parameters had significantly more variation than others but the performance estimates were relatively consistent.

The results of fitting the model parameters to the experimental data are shown in Fig. 17 and Table 2. The performance values predicted by the model matched the performance measurements closely, with a mean absolute error of 3.7 Watts. For comparison, this is less than the advertised measurement error from the power meter.

The results of fitting the model parameters to random subsets of the data are shown in Figs. 18 to 20. Figure 18 shows normalized histograms of the parameter values. Parameters $\tau_{1}, \tau_{2}, k_{1}$, and $k_{2}$ had significantly more variation than the others. Figure 19 depicts integration of the model using those parameter values. This 2-D histogram shows that there is a distribution in the performance predictions, but, except for a few regions, the variation is relatively small. Figure 20 depicts a histogram of the predicted final performance values nine days after the last experimental
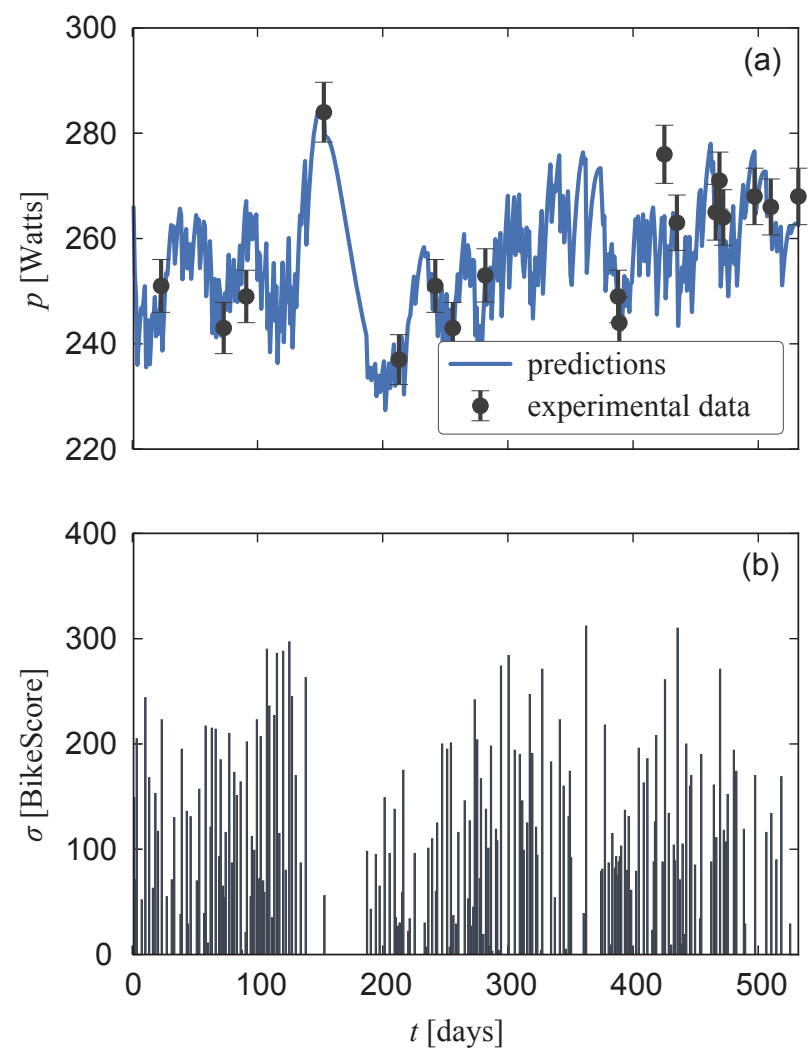

Fig. 17. Plots of (a) the performance data and the predicted performance values using the optimal parameter set found by the genetic algorithm and (b) the experimental training stress measurements

Table 2. Best-fit parameter values corresponding to Fig. 17

\begin{tabular}{ccc}
\hline Parameter & Value & Units \\
\hline$\tau_{1}$ & 77 & ${\text { [Watts }{ }^{\alpha-1}[\text { day }]}^{\beta}$ \\
$\tau_{2}$ & 6.5 & {$[\text { Watts }]^{\beta-1}[$ day $]$} \\
$\alpha$ & 1.3 & - \\
$\beta$ & 0.91 & - \\
$k_{1}$ & 0.19 & ${\text { [Watts }][\text { day }]^{-1}[\text { BikeScore }]^{-1}}$ \\
$k_{2}$ & 0.26 & ${\text { [Watts }][\text { day }]^{-1}[\text { BikeScore }}^{-1}$ \\
$p_{0}$ & 208 & {$[$ Watts $]$} \\
\hline
\end{tabular}



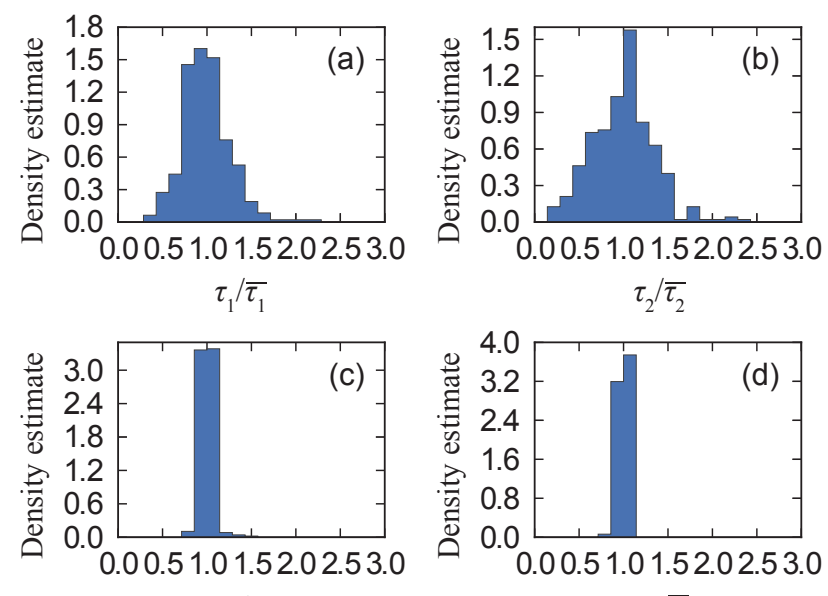

$\alpha / \bar{\alpha}$
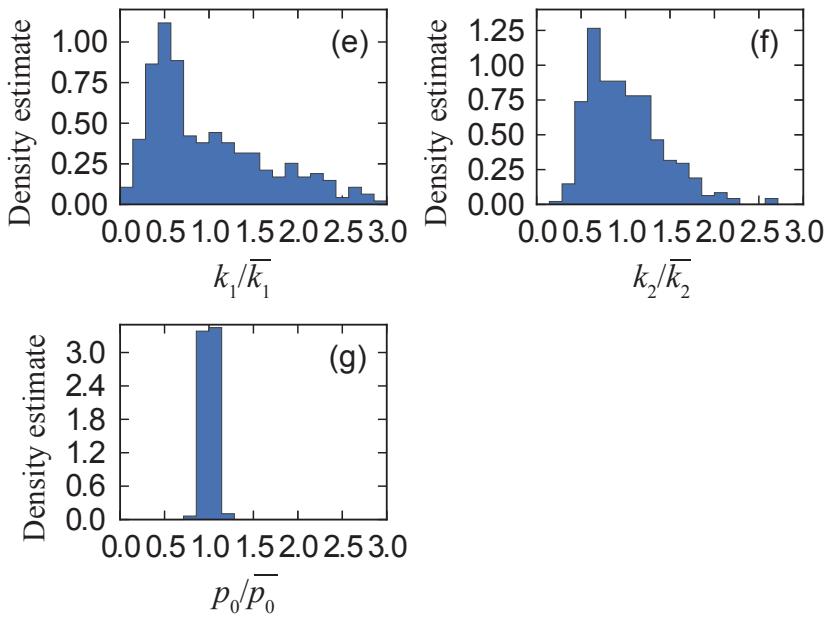

Fig. 18. Density estimation of optimal parameters for randomly selected sets of trials. The horizontal axes were normalized by dividing by the mean parameter value, indicated by an overbar, and the vertical axes were normalized such that the total area under each histogram was 1

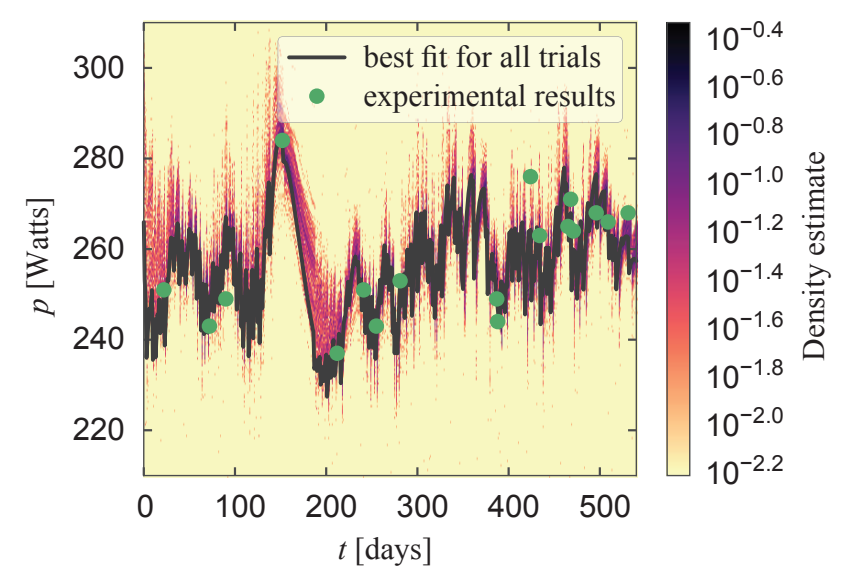

Fig. 19. Normalized 2-D histogram of predicted performance using optimal parameters for randomly selected sets of trials. The counts were normalized such that the sum in each vertical slice was 1. Experimental data and the best fit for all trials are overlaid

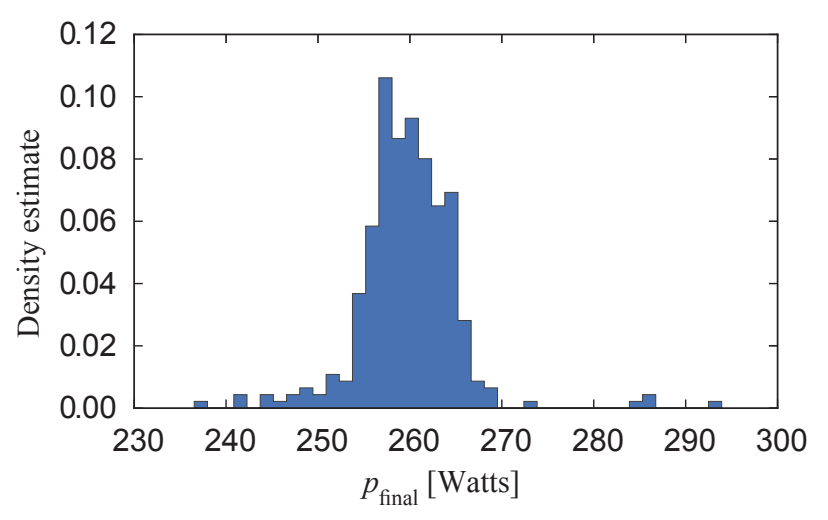

Fig. 20. Density estimation of final performance for randomly selected sets of trials. The vertical axis was normalized such that the total area under the histogram was 1

data point. These estimates are approximately normally distributed with a reasonably small spread.

\section{Discussion}

The results of fitting the model to the data in Fig. 17 are remarkable, given that the model makes predictions over a very long time period of 532 days.

There are a few possible causes of the errors between the performance predictions and the experimental data illustrated in Fig. 17. These include: 1) measurement errors; 2) the athlete exerting an inconsistent effort level; 3) variations in sleep and diet; and 4) limitations in the method of quantifying training stress. While the first two causes of error could easily be reduced by testing in a more controlled environment, the other two causes are phenomena that the model is not designed to capture. For example, variation in sleep and diet could affect the time constants for fitness gain and fatigue recovery, represented in the model as $\tau_{1}$ and $\tau_{2}$, respectively. Similarly, limitations in the stress metric could manifest as variations in the relative influence of stress on fitness and fatigue, i.e. $k_{1}$ and $k_{2}$. We hypothesize that variation in sleep and diet and limitations in the stress metric influenced the relatively large variations in $\tau_{1}, \tau_{2}, k_{1}$, and $k_{2}$ shown in Fig. 18.

The fairly small variation in Figs. 19 and 20 shows that even with some uncertainty in the parameters, fitting to randomly selected experimental trials can produce a relatively consistent final performance. This consistency in final performance predictions is the most important criterion for competitive athletes.

\section{Conclusions}

The nonlinear model presented in Eqs. (1) and (2) successfully captures two essential effects, saturation and 
over-training, which are missed by linear models. As a result, the model can be used to optimize training routines specific to an individual's personal physiological characteristics, constraints, and performance goals.

Simulations for a representative set of parameter values suggest several useful conclusions. First, a taper is necessary to achieve maximum performance on race day since fitness decays at a slower rate than fatigue. Second, multiple solutions exist to achieve optimal average long-term performance, so if an individual is simply trying to maintain a regular schedule without a specific race day in mind, the individual can adjust their training schedule and maintain the same optimal performance. Third, different constraints provide various useful effects that alter the optimal daily training load. Fatigue-based constraints help limit stress during the middle of the training season but are not sufficient at the beginning if the starting fatigue is low. The ATL/CTL ratio and $u / f$ ratio constraints provide a nice progression in training load except at the beginning when fatigue is low. They can also smooth the transition between fitness-based and fatigue-based constraints. A fitness-based constraint can provide a nice progression in training stress, starting from the first day. Finally, a combination of all of these constraints provides the most realistic training strategy that most closely matches conventional wisdom.

After applying the model to historical data and fitting parameters, the results matched the data quite well but also show areas for future research. The results suggest that there are variations in $\tau_{1}, \tau_{2}, k_{1}$, and $k_{2}$ that are not captured by the model; these could be explained by diet, sleep, and limitations in the stress metric, but additional modeling and experimentation are necessary.

It is important to note that the experimental work in this study defined training stress and performance using specific metrics related to cycling. However, the models, training constraints, and algorithms presented in this study can consider a large variety of training stress and performance metrics across a wide range of exercise modes including, but not limited to, running, cycling, and swimming.

The nonlinear model presented in this paper captures important physiological effects missed by previous models, and this gives it new capabilities to design optimal training strategies specifically tailored to individuals' personal physiological characteristics, constraints, and performance goals.

\section{Conflict of interest: Authors state no conflict of interest.}

\section{References}

1. Allen H., A. Coggan (2010) Training and Racing With a Power Meter. VeloPress.
2. Asteroth A., A. Hagg (2015) How to successfully apply genetic algorithms in practice: Representation and parametrization. 2015 International Symposium on Innovations in Intelligent Systems and Applications (INISTA). DOI: 10.1109/INISTA.2015.7276778.

3. Banister E.W., T.W. Calvert, M.V. Savage, T.M. Bach (1975) A systems model of training for athletic performance. Aust. J. Sports Med., 7: 57-61.

4. Busso T. (2003) Variable dose-response relationship between exercise training and performance. Med. Sci. Sports Exerc., 35(7): 1188-1195. DOI: 10.1249/01. MSS.0000074465.13621.37.

5. Busso T., C. Carasso, J.-R. Lacour (1991) Adequacy of a systems structure in the modeling of training effects on performance. J. Appl. Physiol., 71(5): 2044-2049.

6. Busso T., K. Häkkinen, A. Pakarinen, C. Carasso, J.R. Lacour, P.V. Komi, H. Kauhanen (1990) A systems model of training responses and its relationship to hormonal responses in elite weight-lifters. Eur. J. Appl. Physiol. Occup. Physiol., 61: 48-54. DOI: 10.1007/BF00236693.

7. Calvert T.W., E.W. Banister, M.V. Savage, T. Bach (1976) A systems model of the effects of training on physical performance. IEEE Trans. Syst., Man, Cybern., 6(2): 94-102. DOI: 10.1109/TSMC.1976.5409179.

8. Clarke D.C., P.F. Skiba (2013) Rationale and resources for teaching the mathematical modeling of athletic training and performance. Adv. Physiol. Educ., 37(2): 134-152. DOI: 10.1152/advan.00078.2011.

9. Hellard P., M. Avalos, G. Millet, L. Lacoste, F. Barale, J.-C. Chatard (2005) Modeling the residual effects and threshold saturation of training: A case study of Olympic swimmers. J. Strength Cond. Res., 19(1): 67-75. DOI: 10.1519/14853.1.

10. Hellard P., M. Avalos, L. Lacoste, F. Barale, J.-C. Chatard, G.P. Millet (2006) Assessing the limitations of the Banister model in monitoring training. J. Sports Sci., 24(5): 509-520. DOI: 10.1080/02640410500244697.

11. Rao S.S. (2009) Engineering Optimization: Theory and Practice. 4th ed. John Wiley \& Sons. DOI: 10.1002/9780470549124.

12. Schaefer D., A. Asteroth, M. Ludwig (2015) Training plan evolution based on training models. 2015 International Symposium on Innovations in Intelligent Systems and Applications (INISTA). DOI: 10.1109/ INISTA.2015.7276739.

13. Sih B.L., C.H. Negus (2016) Physical training outcome prediction with biomechanics, Part 1: Army physical fitness test modeling. Mil. Med., 181(5S). DOI: 10.7205/ MILMED-D-15-00168.

14. Skiba P.F. (2008) Analysis of power output and training stress in cyclists: The development of the BikeScore ${ }^{\mathrm{TM}}$ algorithm. Tech. rep. PhysFarm Training Systems LLC.

15. Taha T., S.G. Thomas (2003) Systems modeling of the relationship between training and performance. Sports 
Med., 33(14): 1061-1073. DOI: 10.2165/00007256200333140-00003.

16. Thomas L., I. Mujika, T. Busso (2008) A model study of optimal training reduction during pre-event taper in elite swimmers. J. Sports Sci., 26(6): 643-652. DOI: 10.1080/02640410701716782.

17. Thomas L., I. Mujika, T. Busso (2009) Computer simulations assessing the potential performance benefit of a final increase in training during pre-event taper. J. Strength Cond. Res., 23(6): 1729-1736. DOI: 10.1519/ JSC.0b013e3181b3dfa1.

\section{Received 07.03.2017}

Accepted 05.06.2017

(C) University of Physical Education, Warsaw, Poland

Disclosure:

The authors have filed a U.S. provisional patent application (No. 62/328,207) and an international patent application (PCT/US2017/029709) related to the systems and methods for computing and optimizing athletic performance discussed in this paper. 